\title{
Is opium consumption associated with atherosclerotic plaque formation?
}

\author{
Mohammad Saadatnia, M.D. Marzieh Tajmirriahi, M.D. Alireza Zandifar, M.D. \\ Omid Shafaat, M.D. Homa Ebrahimi, M.D.
}

\begin{abstract}
Department of Neurology and Neuroradiology, Isfahan Neurosciences Research Center, Isfahan University of Medical Sciences, Isfahan, Iran.
\end{abstract}

\section{Background and Purpose}

Atherosclerotic plaque formation is related to several risk factors. The effect of opium addiction on the plaque formation is controversial. The aim of this study was to examine the relationship between some risk factors including opium addiction history on the presence of atherosclerotic plaque in the carotid artery.

\section{Materials and Methods}

In this cross-sectional study, we included 220 patients who were screened for brain diseases. B-mode ultrasounographyy was done on bilateral CCAs, carotid bulb, and extracranial internal and external carotid arteries for evaluating the presence of the plaque. Relation between plaque presence and categorical factors were assessed using chi-square test. Multiple logistic regression test was used for adjusting the confounder variables.

\section{Results}

Our data showed the mean of the age and BMI were significantly different between patients with and without plaque $(69.69 \pm 11.04$ vs. $56.22 \pm 13.53, P<0.001$ and $25.80 \pm 3.70$ vs. $27.14 \pm 4.50$ respectively). Chi-square test demonstrated history of diabetes and hypertension were significantly associated with plaque presence (OR: $1.9, \mathrm{Cl}$ : 1.08 - 3.65 and OR: 4.38, $\mathrm{Cl}$ : 2.47 - 7.75), whereas opium consumption and gender were not associated with presence plaque. After adjustments for confounders aforementioned results were confirmed.

\section{Conclusion}

This study demonstrated opium consumption was not associated with the presence of atherosclerotic plaque. More studies with the larger samples size and long followup needed to determine the relationship between opium consumption and atherosclerosis.
Presence of plaque in addicted vs non-addicted

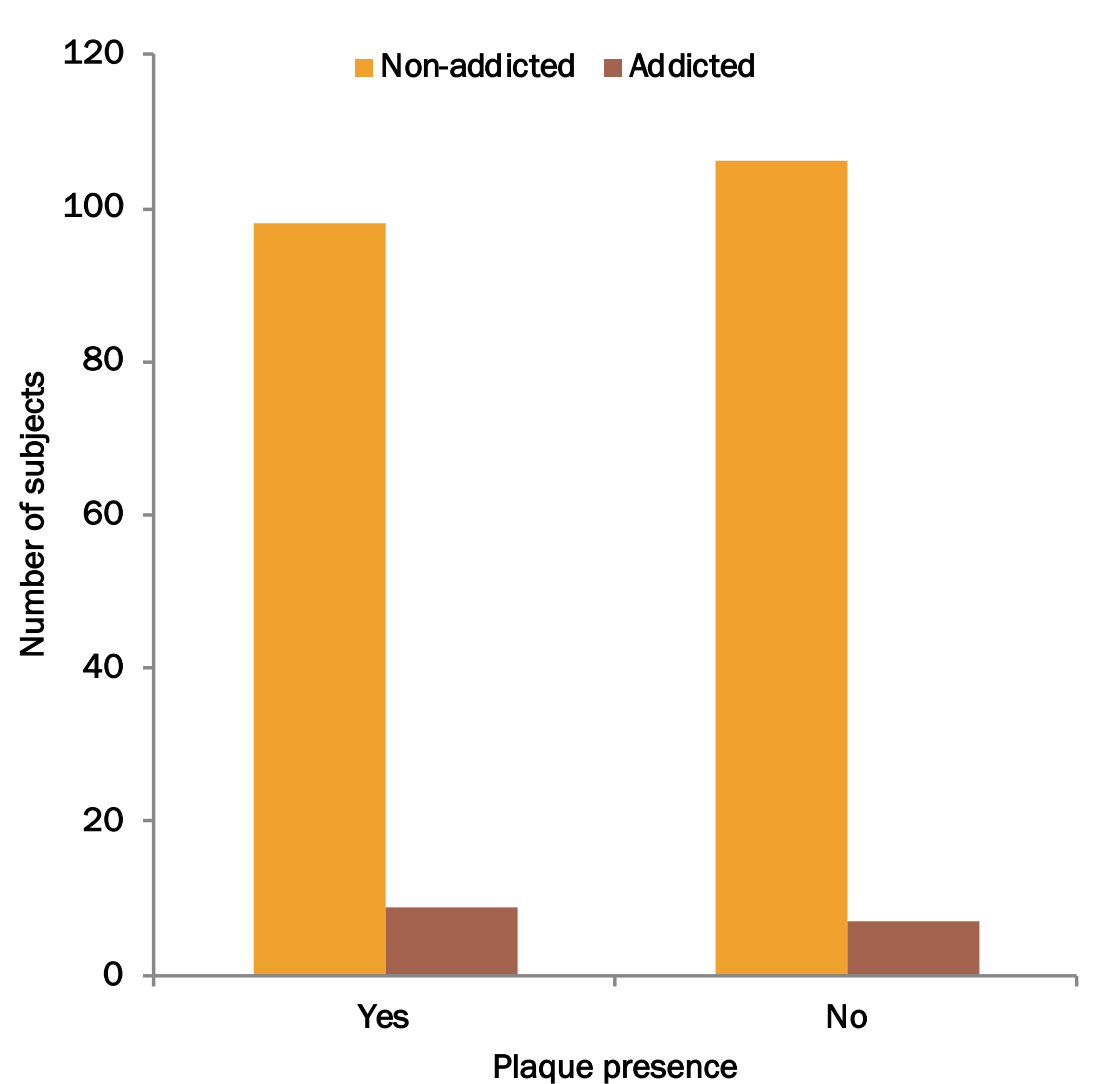

Presence of plaque in diabetic vs non-diabetic subjects

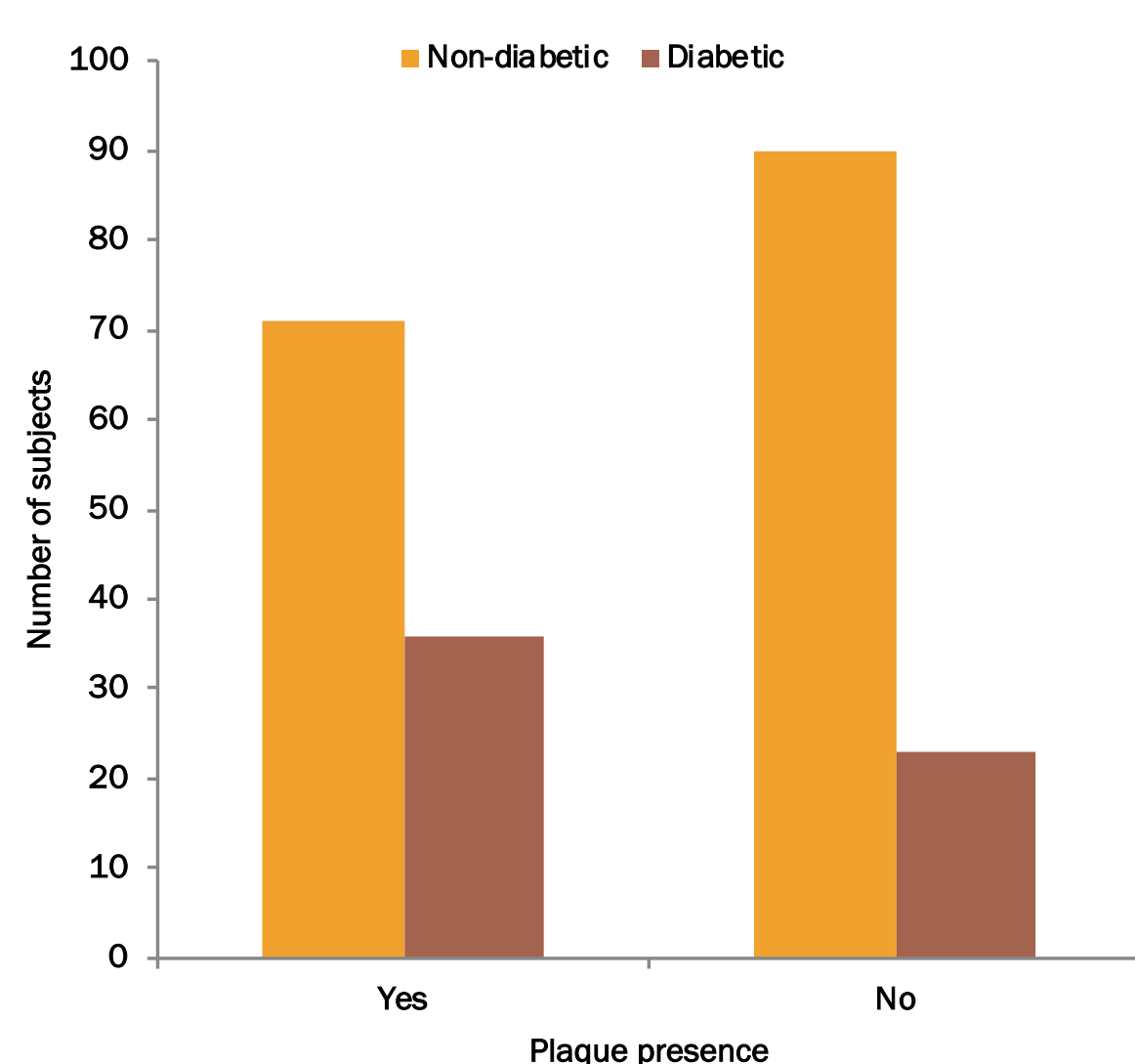

Presence of plaque in patients with HTN vs without HTN

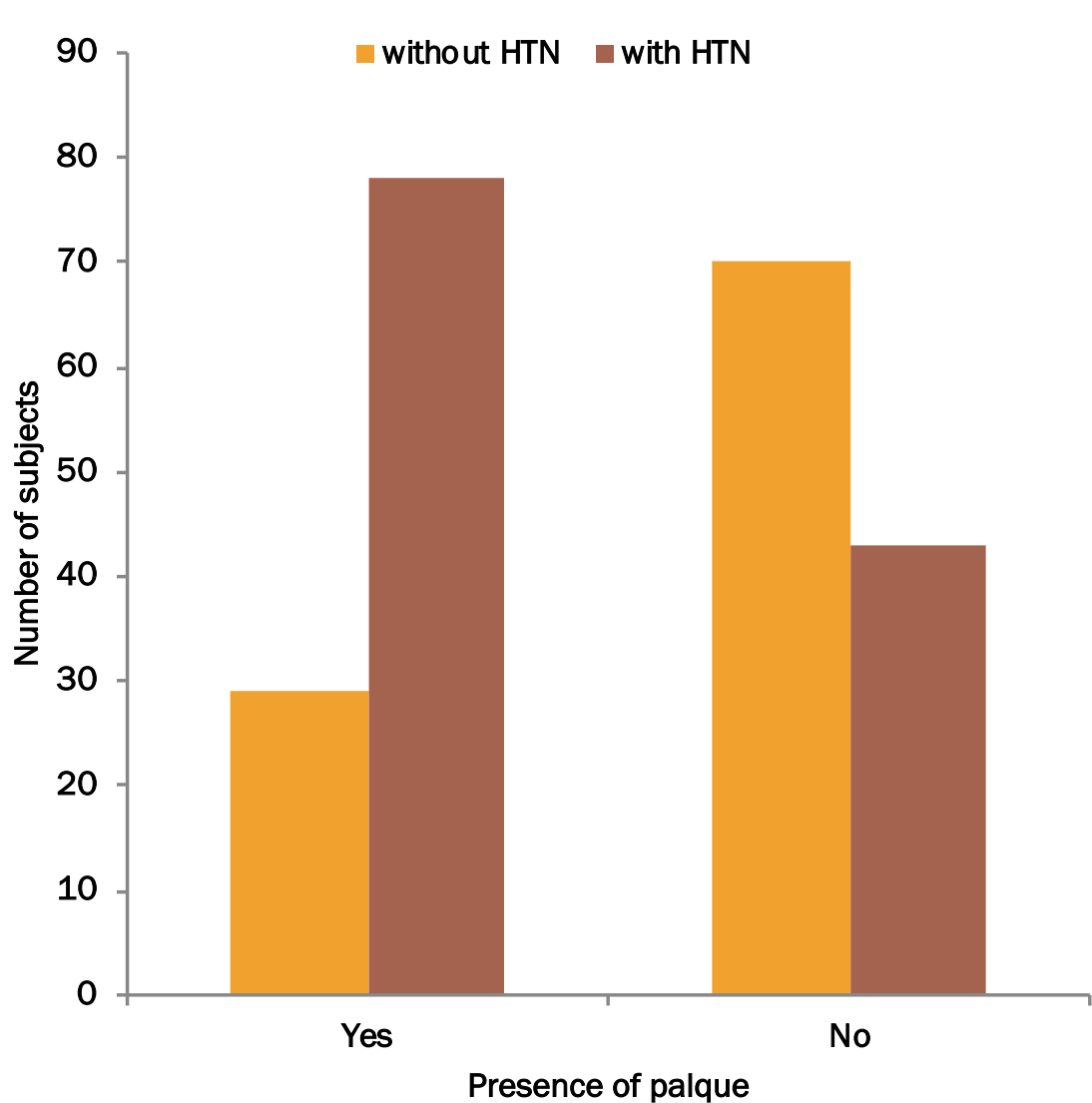

